\title{
Sensor Registration for Robotic Applications
}

\author{
Alen Alempijevic Sarath Kodagoda and Gamini Dissanayake \\ ARC Centre of Excellence for Autonomous Systems (CAS), \\ Faculty of Engineering, \\ University of Technology Sydney, NSW 2007, Australia \\ a.alempijevic, s.kodagoda, g.dissanayake @cas.edu.au
}

Summary. Multi-sensor data fusion plays an essential role in most robotic applications. Appropriate registration of information from different sensors is a fundamental requirement in multi-sensor data fusion. Registration requires significant effort particularly when sensor signals do not have direct geometric interpretations, observer dynamics are unknown and occlusions are present. In this paper, we propose Mutual Information (MI) based sensor registration which exploits the effect of a common cause in the observed space on the sensor outputs that does not require any prior knowledge of relative poses of the observers. Simulation results are presented to substantiate the claim that the algorithm is capable of registering the sensors in the presence of substantial observer dynamics.

\section{Introduction}

With the recent advancements in sensing technologies, it is not unreasonable to assume that sensors will be omnipresent in the near future. Multi-sensor integration and data fusion are essential in order to exploit complementary information from different sensor modalities for achieving robust perception. However, when the relationships between different sensors or sensory signals are unknown, data fusion is not tractable. For example, sensor registration is essential for the fusion of information from a camera mounted in the environment with a mobile robot mounted laser data or fusion of video signals with audio signals. Once the sensor registration is established, multi-sensory data can be fused to perform a variety of tasks in robotics, sensor networks and biomedical engineering.

Mutual information (MI) analysis is well established in the field of medical imaging [1] as a method for image registration and feature selection from a diverse range of sensor modalities. Furthermore, MI has been used as a method to detect statistical relationship or as a measure of coupling between signals [2]. However, traditional MI approaches have a substantial computational cost, in order of a factorial in the number of samples. Fisher et al [3] proposed a methodology for substantially reducing the computational cost of MI. This 
method is based on unsupervised learning of a nonparametric sensing model by maximizing mutual information between signal streams in a lower dimensional space. Experimental results were presented to pin point the mouth of a speaker on a video stream analyzing video and audio signals [5]. This method, however, assumes that there is no relative motion of the speaker in successive image frames. It can not deal with the situations where multiple common sources influence the two sensory signals. These limitations are substantial in most of the robotics applications, which we are embarking on.

In our previous work [6],we have extended the Fisher's idea to incorporate dynamic objects in the environment to establish the sensor registration. Moving targets provide very little instant information at the signal level. However, tracked dynamic targets provide substantial amount of information in the feature level. The target attributes were carefully chosen giving due regards to sensor modality and rate of change of the attribute. The MI based approach was utilized to register the common feature attributes in two sensory signals providing sensor registration. The algorithm was further extended to incorporate the maneuvering targets with possible occlusions as in [10]. However, only stationary observers were considered so far, which is a significant limitation, particularly for robotic applications. In this paper, we have further developed this algorithm to register and calibrate the sensors with moving observers even when target occlusions are present. We do not assume any prior knowledge of relative localization between the observers. The target tracking problem is relatively complex due to the motion of the observer, maneuvering dynamics of the target and occlusions. Multiple model approach, in particular the Interacting Multiple Model (IMM) [7], provides one of the most effective frameworks for tracking maneuvering targets. Sequential Probability Ratio Test (SPRT) is used to eliminate false tracks that get initiated due to anomalies in the background and spurious data [8].

The paper is organized as follows. Section 2 discusses the Fisher's MI based approach for sensor registration. In Section 3, the problem of tracking maneuvering targets with moving observers is formulated. IMM with SPRT is discussed in Section 4. Simulation results are presented in Section 5. Section 6 concludes the paper providing future direction of the research.

\section{Mutual Information based Sensor Registration}

In this section we describe an information theoretic approach for sensor registration, which is based on [3]-[5]. One important aspect of this method is it does not assume any prior information about relationships among either sensory signals or sensors.

Fisher's Mutual Information Algorithm: Let $X^{a} \in \Re^{N_{a}}$ and $X^{b} \in$ $\Re^{N_{b}}$ are two high dimensional random variables. Those are passed through functions $Y^{a}=g\left(X^{a}, H_{a}\right)$ and $Y^{b}=g\left(X^{b}, H_{b}\right)$ where $Y^{a} \in \Re^{M_{a}}, Y^{b} \in \Re^{M_{b}}$ with $M_{a}<<N_{a}$ and $M_{b}<<N_{b}$. Although the mapping can be done through 
any differentiable function, here, $H_{a}$ and $H_{b}$ are treated as coefficients of linear projections. The idea is now to choose the projection coefficients to optimize the MI, which is,

$$
I\left(Y^{a} ; Y^{b}\right)=h\left(Y^{a}\right)+h\left(Y^{b}\right)-h\left(Y^{a}, Y^{b}\right)
$$

where, $h($.$) is the differential entropy, which can be defined for a random$ variable $Y$ with density $p_{Y}$ as,

$$
h(Y)=-\int_{\Omega} p_{Y}(y) \log \left(p_{Y}(y)\right) d y
$$

Our goal is to maximize the mutual information between two random variables $Y^{a}$ and $Y^{b}$. This can be achieved by maximizing the entropies of $Y^{a}$ and $Y^{b}$ and minimizing the joint entropy, $h\left(Y^{a}, Y^{b}\right)$. Entropy is maximum in a uniform distribution, which is therefore chosen as the desired distribution in the transformed space. The density of the samples are estimated using Parzen density estimator [11]. (2) is linearized with a second order Taylor series expanded around the uniform distribution and the difference between entropies of desired and estimated densities are calculated. The updating term now becomes [4],

$$
\begin{gathered}
\Delta y_{i}^{(k)}=b_{r}\left(y_{i}^{(k-1)}\right)-\frac{1}{N} \sum_{j \neq i} \kappa_{a}\left(y_{i}^{(k-1)}-y_{j}^{(k-1)}, \sum\right) \\
b_{r}\left(y_{i}\right)_{j} \approx \frac{1}{d}\left(\kappa\left(y_{i}+\frac{d}{2}, \sum\right)_{j}-\kappa\left(y_{i}-\frac{d}{2}, \sum\right)_{j}\right) \\
\kappa_{a}\left(y, \sum\right)=\kappa\left(y, \sum\right) * \kappa^{\prime}\left(y, \sum\right) \\
\kappa_{a}\left(y, \sum\right)=-\left(2^{M+1} \pi^{M / 2} \sigma^{M+2}\right)^{-1} \exp \left(-\frac{y^{T} y}{4 \sigma^{2}}\right) y
\end{gathered}
$$

where, $\kappa_{a}($.$) is a kernel: a Gaussian pdf is assumed here. y_{i}$ symbolize a sample of either $Y^{a}$ or $Y^{b}, M=M_{a}, M_{b}$ or $M_{a}+M_{b}$ based on the term of (1) that is considered. The $j^{\text {th }}$ element of $b_{r}\left(y_{i}\right)$ is defined as $b_{r}\left(y_{i}\right)_{j} . d$ is the support of the output space. $N$ is the number of samples. The adaptation procedure includes the update rule (3) followed by a least squares solution for $h_{a}$ and $h_{b}$ until a maximum is reached. The stopping criteria used is,

$$
\delta=\frac{\max \left(\Delta_{N N}\right)-\min \left(\Delta_{N N}\right)}{\max (\Delta)}
$$

where, the term $\Delta_{N N}$ is the nearest neighbour distance in the resulting output distribution, $\max ($.$) and \min ($.$) are the maximum distance and mini-$ mum distance between any two points in the output space. The numerator is a measure of uniformity of the output space and the denominator is a measure of how well the output space is filled. 


\section{Process and Observation Models for Tracking with Moving Observers}

In this section, we formulate the tracking problem of maneuvering targets with a moving observer. When the observer is stationery, the targets tracked in the respective sensory spaces can be used to calculate necessary attributes to be used with MI. However, if the observers are moving, the attributes calculated on the tracked objects in the sensory spaces is correlated to the observer movement. This may cause the same target's attributes calculated from different moving observers to be non informative resulting in the MI based sensor registration to fail. This problem can be solved, if the targets are being tracked in their respective locally defined world frames. Here, we assume the observer localization to a known uncertainty using some external means (eg. SLAM) in these local coordinate frames. However, we do not assume any knowledge of the relative location of the observers.

\subsection{Process Model}

The target dynamics in the world coordinates, W, (Fig.1(a)) are modelled by a combination of constant speed and turn rate models.

$$
\dot{\mathbf{x}}_{w}(t)=\mathbf{f}\left(\mathbf{x}_{w}(t), \omega, \boldsymbol{\nu}(t), t\right)
$$

where, $\dot{\mathbf{x}}_{w}(t)=[x, \dot{x}, y, \dot{y}],\{x, y\}$ are the position coordinates of the target in $x$ and $y$ directions and $\{\dot{x}, \dot{y}\}$ are the respective velocities in the world coordinates. $\omega$ is the turn rate of the target.

\subsection{Observation Model}

Here we assume range and bearing observations. Therefore the observation model in the sensory space is,

$$
\mathbf{z}(t)=\mathbf{h}\left(\mathbf{x}_{w}(t), \mathbf{x}_{s}(t), \varpi(t), t\right)
$$

where, $\mathbf{z}(t)=\left[\begin{array}{ll}r & \theta\end{array}\right]^{T}$ are range and bearing to the target in the sensory space. $\mathbf{x}_{s}(t)$ is the pose of the observer in the world reference frame. $\varpi(t)$ is the measurement noise (zero mean Gaussian).

\section{Tracker Based on Interacting Multiple Model (IMM)}

One of the best algorithms to track maneuvering targets with stationary/moving observers is the IMM algorithm. In the following, the IMM is used to track maneuvering targets whilst SPRT is used for track initiation, maintenance and deletion. Once the targets are detected and tracked, the attributes of the 


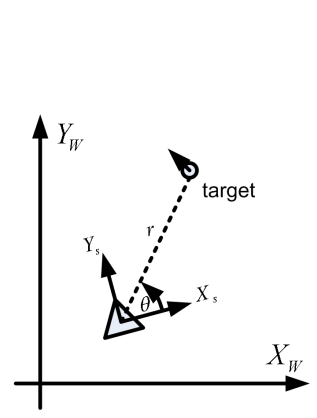

(a)

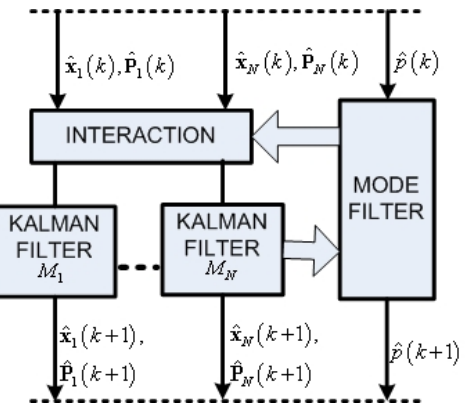

(b)

Fig. 1. a) Coordinate frames: $W$ - world coordinates, $S$ - sensor coordinates and b) The IMM algorithm [10]: $\hat{\mathbf{x}}_{i}, \hat{\mathbf{P}}_{i}$ define the states and their covariance matrices for $i=1, \ldots ., N$ hypotheses. $\hat{p}(t)$ is the mode probability

targets are calculated and used in MI based sensor registration. The IMM algorithm [7] is shown in the Fig.1(b).

False tracks may be initiated due to spurious target detections. The true track confirmation and false track deletion are handled using the ideas from integrated probabilistic data association (IPDA) [9] with SPRT [8]. Using the Markov relationship, the probability of existence of the true target, $P_{T}(k+1 \mid k)$ before the receipt of data in scan $k+1$ is [8],

$$
P_{T}(k+1 \mid k)=P_{22} P_{T}(k \mid k)+P_{12}\left[1-P_{T}(k \mid k)\right]
$$

where $P_{22}$ is the probability of transition from observable state to observable state, whilst $P_{12}$ is the probability of transition from unobservable state to observable state. Then, the update of the probability of target existence is $[9]$,

$$
P_{T}(k+1 \mid k+1)=\frac{1-\delta_{k+1}}{1-\delta_{k+1} P_{T}(k+1 \mid k)} P_{T}(k+1 \mid k)
$$

where $\delta_{k+1}$ is defined as,

$$
\delta_{k+1}= \begin{cases}P_{D} P_{G} & N_{k+1}=0 \\ P_{D} P_{G}\left[1-\bar{V} \sum_{i=1}^{N_{k+1}} \frac{1}{P_{G}(2 \pi)^{M / 2} \sqrt{|S(k+1 \mid k)|}} e^{-d_{i}^{2} / 2}\right] & \text { Otherwise }\end{cases}
$$

$P_{D}$ is the probability of detection, $P_{G}$ is the gate probability, $V_{G}$ is the gate volume, $N_{k+1}$ is the number of measurements inside the validation gate, $S$ is the innovation covariance, and $d_{i}^{2}$ is the normalized innovation squared of the $i^{t h}$ measurement. The Log Likelihood Ratio, LLR, can now be defined as [8], 


$$
L L R_{k+1}=\ln \left(\frac{P_{T}}{1-P_{T}}\right)
$$

Once the LLR is obtained, track confirmation and termination thresholds are determined using the SPRT [8] as,

$$
\begin{gathered}
L L R_{k+1} \geq \ln \left(\frac{1-\beta_{T}}{\alpha_{T}}\right), \text { declare track confirmation } \\
\ln \left(\frac{\beta_{T}}{1-\alpha_{T}}\right)<L L R_{k+1}<\ln \left(\frac{1-\beta_{T}}{\alpha_{T}}\right), \text { continue test } \\
L L R_{k+1} \leq \ln \left(\frac{\beta_{T}}{1-\alpha_{T}}\right), \text { delete track }
\end{gathered}
$$

where, $\alpha_{T}$ is the probability of false track confirmation, and $\beta_{T}$ is the probability of true track termination.

\section{Simulation Results}

Simulations are carried out for signal level and feature level sensor registration. The results are presented bellow.

\subsection{Signal Level Sensor Registration}

The MI algorithm was initially evaluated through simulations. Twenty two different signals were generated and four random signals from the set were supplied to sensor 1 and another four to sensor 2 with only one common signal (signal 1 in sensor 1 and signal 3 in sensor 2 in Fig.2. Calculation of Mutual Information was performed on 80 samples imposing the following criteria on the variation of $\delta$. Firstly the convergence limit must be less than 0.085 , convergence variation contained in a 0.001 limit and a minimal convergence length of 10 iterations. It is to be noted that the convergence criterion is dependant on the number of samples obtained from the signal and (4). With the imposed criteria, a match was found in 28 iterations (Fig.2(b)).

Table 1 shows the calculated MIs with the highest MIs are denoted by symbol * The algorithm correctly identifies common signals between sensors as signal 1 of sensor one and signal 3 of sensor two, which agrees with the simulation.

Table 1. MI values

\begin{tabular}{lllll}
\hline Sensor/Signal 1 & 2 & 3 & 4 \\
\hline 1 & $0.7744^{*}$ & 0.1050 & 0.0085 & 0.0490 \\
2 & 0.1596 & 0.1124 & $0.7372^{*}$ & 0.1758 \\
\hline
\end{tabular}




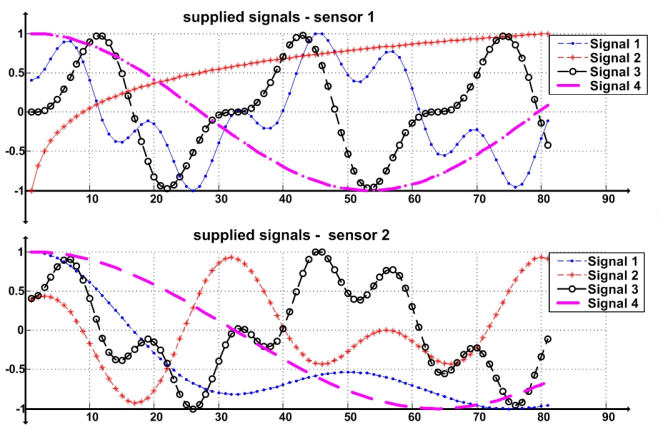

(a)

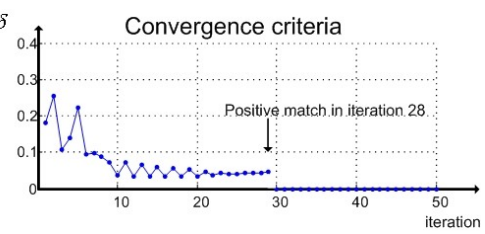

(b)

Fig. 2. Signal level sensor registration a) Simulated signal streams and b) MI learning curve

\subsection{Sensor Registration based on Moving Observers and Moving Targets}

The targets can be tracked in the sensory space and attributes can be estimated even with moving observers. However, this introduces correlations of the observer motion in the estimated target attributes. Since the observers have independent dynamics, the attributes are not mutually informative. Therefore, the targets have to be tracked in a locally defined world reference frames. Fig.3(a) shows a simulation study carried out with two moving observers (O1 and O2) and three maneuvering targets (T1, T2 and T3). Fig.3(b) shows the extracted target attributes in respective observers' locally defined world coordinates. The MI based algorithm converges at 39 th iteration as depicted in Fig.3(c), detecting the most informative attribute as the speed of Target 2 (Table 2 ). The path of Target 2 in the two local world co-ordinate frames is then used to compute the relative calibration between the sensors through a least square fit. The tracked target poses in observer 2 can now be transformed onto the locally defined world reference frame of observer 1 (Fig.3(d)). It is to be noted that the target tracks of both observers are overlapping, confirming correct sensor registration.

Table 2. MI values: a-angle, s-speed, r-range, T-target

\begin{tabular}{llllllllll}
\hline Sensor/Signal a.T1 & a.T2 a.T3 & s.T1 & s.T2 & s.T3 & r.T1 & r.T2 & r.T3 \\
\hline 1 & 0.9 & 50 & 15 & 0.06 & $78^{*}$ & 0.2 & 0.1 & 0.2 & 0.1 \\
2 & 19.6 & 22 & 3.8 & 1.9 & $100^{*}$ & 0.5 & 1.5 & 1 & 0.9 \\
\hline
\end{tabular}

The main requirement of this sensor registration strategy is that at least one common cause should be observed by the both observers. Any number 


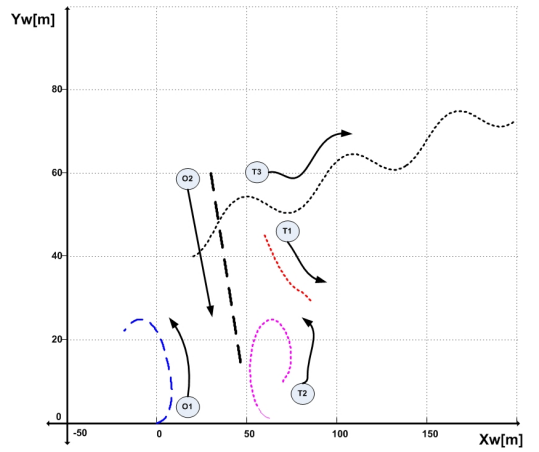

(a)

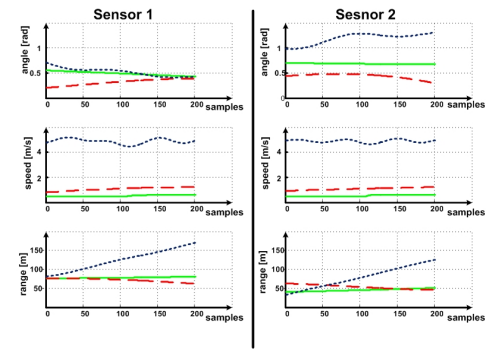

(b)

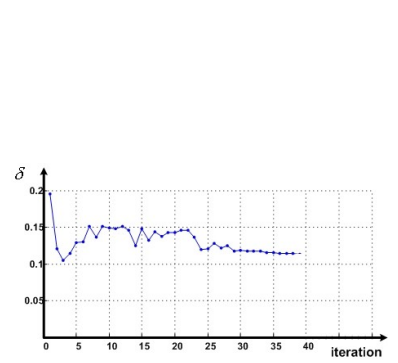

(c)

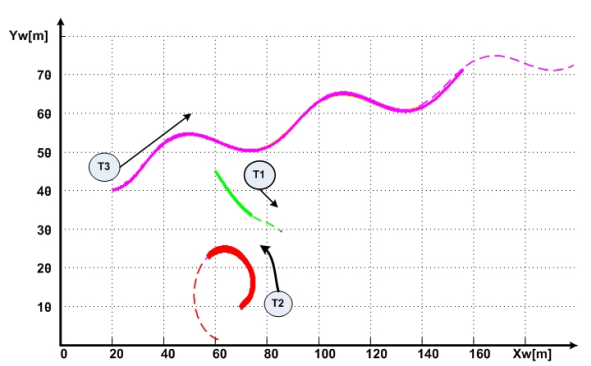

(d)

Fig. 3. Sensor registration with moving observers and moving targets (a) Simulation environment: O - observer, T - target, (b) Extracted target attributes, solid linetarget 1, dashed line - target 2, dotted line - target 3, (c) Learning curve, and (d) All data transformed into sensor 1 reference frame

of targets that influence only one sensor may be present. Simulation in Fig.4 is used to demonstrate this scenario and the effect of target occlusion. Two moving observers (O1 and $\mathrm{O} 2$ ) and five maneuvering targets (T1 to T5) are present. Observer $\mathrm{O} 1$ can see targets $\mathrm{T} 1, \mathrm{~T} 2$ and $\mathrm{T} 3$ while observer $\mathrm{O} 2$ can see targets T1, T4 and T5. Occlusions were introduced at inset 1 and 2 in Fig.4(d). Fig.4(b) shows the extracted target attributes in a locally defined world coordinates. Two hundred samples of the target attributes were used in the MI based algorithm, which converged at iteration 48 as depicted in Fig.4(c), resulting in the angle to target 1 being the most informative (Table 3). Fig.4(d) depicts that the observer 2 data transformed on to the observer 1 are aligned with each other, which shows that the sensor registration is correct. This confirms that MI based algorithm correctly uses common causes rather than distracted by other targets. While the targets are occluded, the IMM estimates their pose. Therefore, the algorithm is also immune to target occlusions. 


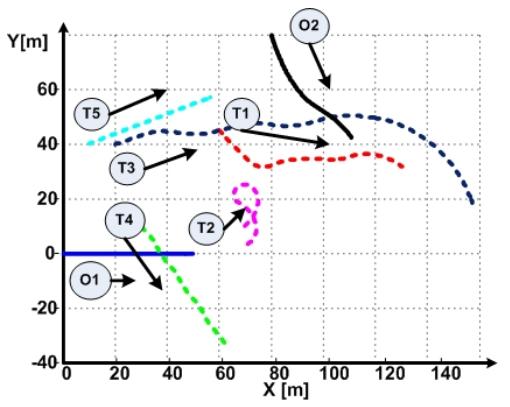

(a)

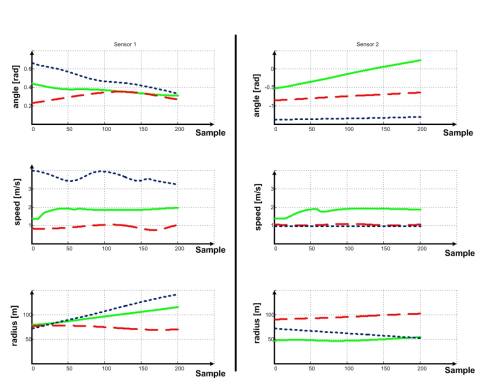

(b)

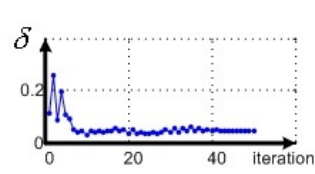

(c)

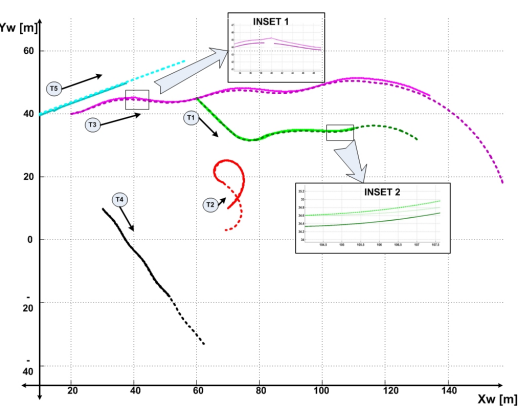

(d)

Fig. 4. Sensor registration with moving observers and moving targets experiencing occlusions (a) Simulation environment: O - observer, T - target, (b) Extracted target attributes, solid line- target 1, dashed line - target 2, dotted line - target 3, (c) Learning curve, and (d) All data transformed into sensor 1 reference frame

Table 3. MI values: a-angle, s-speed, r-range, T-target

\begin{tabular}{|c|c|c|c|c|c|c|c|c|c|}
\hline Sensor/Signal & l a.T1 & a.T $2 /$ & Г4 а.Т3/T & 5 s.T 1 & 1 s.T2/ & T4 s.T3/T: & 5 r.T1 & r.T2/ & /T4 r.T3/T5 \\
\hline 1 & $18.2^{*}$ & 12 & 8.2 & 2.5 & 0.9 & 0.2 & 0.9 & 0.8 & 0.9 \\
\hline 2 & $87^{*}$ & 21 & 36.9 & 1.2 & 1.1 & 32.5 & 1.2 & 1.1 & 2.4 \\
\hline
\end{tabular}

\section{Conclusions and Future Work}

One of the main requirements of multi-sensor fusion is sensor registration. In mobile robotics applications, sensor registration is complicated due to the presence of moving observers and occlusions, and the dynamic nature of the environment. Under these conditions signal level information provided by a common cause can not be easily interpreted. Therefore, we proposed to track the dynamic targets in the environment and use their attributes as the informative signals in a MI based approach for sensor registration. The target tracking problem is nontrivial due to the unknown dynamics of the targets, 
false detections, and occlusions. These problems were successfully handled by IMM, and SPRT. Then the MI based algorithm was successfully used for sensor registration. However, one of the deficiencies of the current algorithm is that it requires high computational effort as it requires the accumulation of a large sample set to perform the sensor registration. Improving computational efficiency to achieve real time operation is the subject of our future work.

Acknowledgements: This work is supported by the ARC Centre of Excellence programme, funded by the Australian Research Council (ARC) and the New South Wales State Government.

\section{References}

1. Pluim J P W, Maintz J B A, Viergever M A (2003), Mutual-information-based registration of medical images, a survey, IEEE Transactions on Medical Imaging, vol. 22: 986-1004

2. Pompe B, Blidh P, Hoyer D, Eiselt M (1998), Using mutual information to measure coupling in the cardiorespiratory system, IEEE Engineering in Medicine and Biology Magazine, vol. 17: 32-39.

3. Fisher III J W, Principe J C (1997), Entropy manipulation of arbitrary nonlinear mappings, Proceedings of the IEEE Workshop on Neural Networks for Signal Processing: 14-23.

4. Fisher J, Wainwright M J, Sudderth E B, Willsky A S (2002), Statistical and Information-Theoretic Methods for Self-Organization and Fusion of Multimodal, Networked Sensors, International Journal of High Performance Computing Applications, vol. 16: 337-353

5. Fisher III J W, Darrell T (2004), Speaker association with signal-level audiovisual fusion, IEEE Transactions on Multimedia, vol. 6: 406-413

6. Alempijevic A, Kodagoda S, Underwood J, Kumar S, Dissanayake G (2006), Mutual Information based Sensor Registration and Calibration, Proceedings of the IEEE/RSJ International Conference on Intelligent Robots and Systems (IROS 2006), Beijing: 25-30

7. Blom H A P, Bar-Shalom Y (1988), The interacting multiple model algorithm for systems with Markovian switching coefficients, The IEEE Transactions on Automatic Control, Vol.33, No. 8: 780-783.

8. Blackman S, Popoli R (1999), Design and Analysis of Modern Tracking Systems, Boston, Artech House

9. Musicki D, Evans R, Stankovic S (1994), Integrated probabilistic data association, IEEE Transactions on Automatic Control, Vol. 39, No. 6: 1237-1241

10. Kodagoda K R S, Alempijevic A, Underwood J, Kumar S, Dissanayake G (2006), Sensor Registration and Calibration using Moving Targets, Proceedings of nineth International Conference on Control, Automation, Robotics and Vision (ICARCV2006): 830-835

11. Parzen E (1962), On estimation of probability density function and mode, Annals of Mathematical Statistics, 33: 1065 - 1076 